\title{
Nonlinear Simulation of Collapse Phenomenon in Helical Plasma with a Large Pressure Gradient
}

\author{
Naoki MIZUGUCHI ${ }^{1,2)}$, Yasuhiro SUZUKI ${ }^{1)}$ and Nobuyoshi OHYABU ${ }^{1,2)}$ \\ ${ }^{1)}$ National Institute for Fusion Science, Toki 509-5292, Japan \\ ${ }^{2)}$ The Graduate University for Advanced Studies (SOKENDAI), Toki 509-5292, Japan
}

(Received 16 November 2007 / Accepted 26 February 2008)

\begin{abstract}
Nonlinear magnetohydrodynamics (MHD) simulations are conducted for a helical plasma with a large pressure gradient to investigate the collapse event induced by MHD instabilities. The simulation results show that the ballooning-like instabilities on an intermediate spatial scale induce disordering of structures in the barrier region and a drop in the central pressure. It was revealed that the core pressure fall is related to disordering of the magnetic field structure. The simulation results are compared qualitatively with the experimental observations of the collapse events in the superdense core state of the Large Helical Device (LHD). Compared with our previous simulation studies of similar situations in the spherical tokamak (ST) plasma, the results for the helical cases show milder collapses than those in the ST case.
\end{abstract}

(c) 2008 The Japan Society of Plasma Science and Nuclear Fusion Research

Keywords: magnetohydrodynamics, collapse, helical system, simulation, superdense core

DOI: $10.1585 /$ pfr.3.S1034

\section{Introduction}

In recent experiments in the Large Helical Device (LHD), high performance confinement with a superdense core (SDC) inside the internal diffusion barrier (IDB) has been achieved using the pellet injection scheme [1]. In the re-heating stage in such a discharge, where the plasma beta increases gradually after ceasing the pellet injection, an abrupt flushing of the central density occurs in some cases. Such a collapse phenomenon, which is named "core density collapse" (CDC) [2,3], should be avoided since it often limits the density increase. Although the physical mechanism of CDCs remains unclear, several models based on the magnetohydrodynamic (MHD) instability or the equilibrium limit have been proposed. Revealing the mechanism of CDC is one of the key issues in further development of the confinement toward the high-density branch.

This article reports the first attempt to conduct a nonlinear MHD simulation in helical plasma with a large pressure gradient, which corresponds to the IDB-SDC state of LHD. The result obtained by the simulation would provide us with basic understandings for the collapse phenomena, including the $\mathrm{CDC}$, from the viewpoint of MHD.

\section{Simulation Model}

We solve the time development of the standard set of compressive, resistive, nonlinear MHD equations

$$
\frac{\partial \rho}{\partial t}=-\nabla \cdot(\rho v)
$$

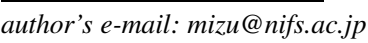

$$
\begin{aligned}
\frac{\partial}{\partial t}(\rho \boldsymbol{v})= & -\nabla \cdot(\rho \boldsymbol{v} \boldsymbol{v})-\nabla p+\boldsymbol{j} \times \boldsymbol{B} \\
& +\mu\left(\nabla^{2} \boldsymbol{v}+\frac{1}{3} \nabla(\nabla \cdot \boldsymbol{v})\right), \\
\frac{\partial \boldsymbol{B}}{\partial t}= & -\nabla \times \boldsymbol{E}, \\
\frac{\partial p}{\partial t}= & -\nabla \cdot(p \boldsymbol{v})-(\gamma-1)\left(p \nabla \cdot \boldsymbol{v}+\eta \boldsymbol{j}^{2}\right),
\end{aligned}
$$

in a full-toroidal three-dimensional geometry. The variables, $\rho, \boldsymbol{v}, \boldsymbol{B}$, and $p$ represent the mass density, the fluid velocity, the magnetic field, and the pressure, respectively. The current density $\boldsymbol{j}$ and the electric field $\boldsymbol{E}$ are calculated from

$$
\begin{aligned}
\boldsymbol{j} & =\nabla \times \boldsymbol{B}, \\
\boldsymbol{E} & =-\boldsymbol{v} \times \boldsymbol{B}+\eta \boldsymbol{j} .
\end{aligned}
$$

In (1)-(6), the dissipation terms are included as the resistivity $\eta$ and the viscosity $\mu$. These terms are assumed to be uniform constants for simplicity. The Ohmic heating term is evaluated in the evolution of the pressure $p$, whereas the viscous heating is ignored for simplicity. All the spatial derivatives are expressed numerically using the fourthorder central difference scheme. The time integration is solved using the fourth order Runge-Kutta method.

To follow the geometry of the helical devices with continuously wound magnetic coils, we adopt the helicaltoroidal coordinate system used in the HINT code [4]. This coordinate system $\left(u^{1}, u^{2}, u^{3}\right)$ uses a rectangular grid $\left(u^{1}, u^{2}\right)$ in the poloidal cross section and a toroidal grid $\left(u^{3}\right)$ with geometrically regular intervals. With the poloidal angle $\theta$, toroidal angle $\phi$, and the minor radius $r,\left(u^{1}, u^{2}, u^{3}\right)$ 
is described as [4]

$$
\begin{aligned}
& u^{1}=r \cos (\theta-h \phi), \\
& u^{2}=r \sin (\theta-h \phi), \\
& u^{3}=-\phi,
\end{aligned}
$$

where the number of helical period $h$ is $10 / 2$ to follow the LHD configuration. The boundary condition of the computation for the $u^{1}$ and the $u^{2}$ directions is the perfect conductor condition, and that for the $u^{3}$ direction assumes the periodicity of $2 \pi$. The number of the numerical grids is $\left(N_{1}, N_{2}, N_{3}\right)=(62,146,500)$.

The initial condition for the simulation is given by the numerical solution of the HINT2 code, which models an average experimental configuration of LHD [5]. The simulation geometry includes the region out of the separatrix. To treat such an external region continuously from the inside of the separatrix, we artificially add a small uniform value to the pressure initially.

The simulation starts by adding tiny random perturbations to the velocity component of the initial equilibrium. Then, the spontaneous time development of the MHD system is solved by vector-parallel calculation on a supercomputer.

\section{Simulation Result}

The MHD equations (1)-(6) are solved in a dimensionless form by normalizing all the variables with characteristic values, such as $1 \mathrm{~m}$ for the spatial scale, and $1 \mathrm{~T}$ for the magnetic field. Uniform density $\rho=1$ is assumed for the initial condition for simplicity. Uniform constants for the dissipation coefficients $\eta=10^{-4}$ and $\mu=10^{-3}$ are used throughout this study to satisfy numerical stability and accuracy for the behavior of interest. It should be noted that the values of these terms used are much larger than the classical ones, $\eta_{\mathrm{c}} \sim 10^{-8}-10^{-7}$, and $\mu_{\mathrm{c}}$ should be negligible for the MHD approximation. Therefore, several aspects of the simulation results might be different from the classical responses, such as the growth rate of the resistive instabilities and the instantaneous response for very small scale behavior. The unit of time, i.e., the Alfvèn transit time $\tau_{\mathrm{A}}$ corresponds to the order of $1 \mu \mathrm{sec}$ for the standard parameters of the LHD experiments. For the main results described in this article, the initial equilibrium, which roughly models the IDB-SDC state of LHD just before the CDC events, is used. Its pressure profile is shown in Fig. 1 (case A). The maximum and the volume averaged beta value are $\beta_{0}=6.6 \%$ and $\langle\beta\rangle=1.8 \%$, respectively. Compared with the standard high-beta discharges of LHD experiments (case $\mathrm{C}$ ), the pressure gradient in the barrier region is markedly large due to the large Shafranov shift.

Figure 2 shows the time evolution of the kinetic energy after solving the linearized system of (1)-(6). One can see a linear growth of the instability at $t>50 \tau_{\mathrm{A}}$ for case A. In Fig. 2, the trace for the case using a lower beta equilibrium with $\beta_{0}=2.5 \%$ (case $\mathrm{B}$ ) is also plotted for

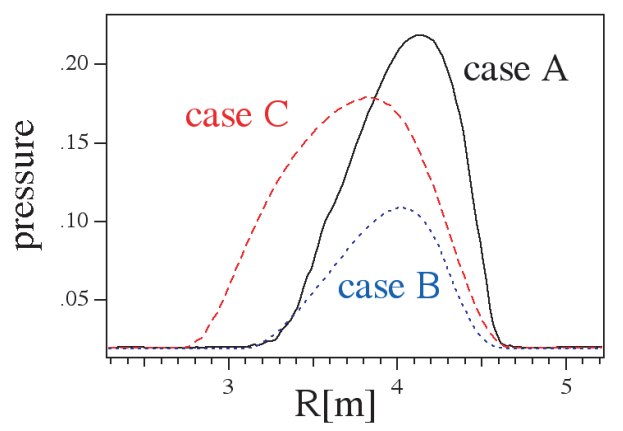

Fig. 1 Radial pressure profiles of the initial equilibria for the case $\mathrm{A}, \mathrm{B}$, and $\mathrm{C}$ on the horizontally elongated cross section.

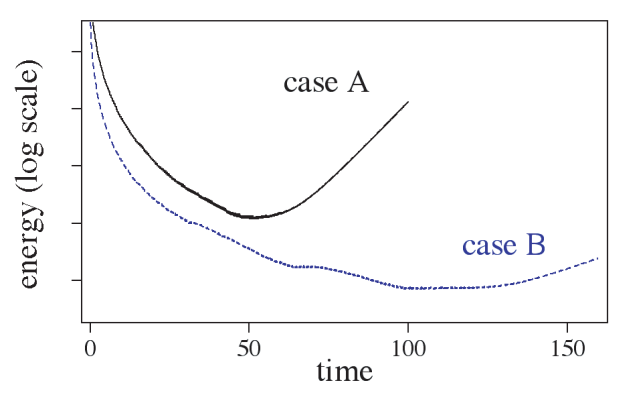

Fig. 2 Time development of kinetic energy for the linear simulation.

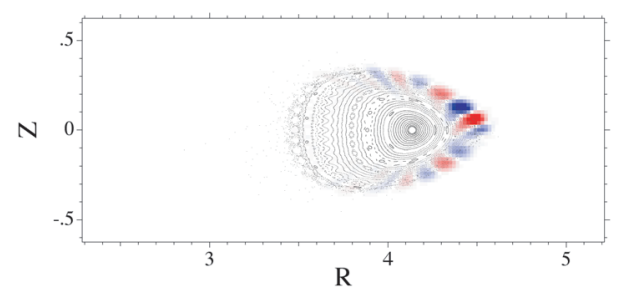

Fig. 3 Poloidal mode structure of the perturbations in the pressure on the horizontally elongated cross section.

reference. One can see that the growth rate for case $A$ is much larger than that for case B. In addition, the resistivity scans show a positive dependence of the growth rate on the resistivity, which proves the modes to be the resistive ones. These modes have intermediate poloidal mode numbers $m \sim 10$. The poloidal mode structures, shown in Fig. 3, clearly exhibit the ballooning nature in that the fluctuations are localized in the outer region.

The nonlinear long-term evolution of the energy and the maximum pressure is shown in Fig. 4. One can see that the growth and saturation of the energy repeats three times before reaching a relaxed state. The growth shown in Fig. 2 corresponds to the first one $\left(t<100 \tau_{\mathrm{A}}\right)$. During this relaxation process, the plasma changes its shape gradually. The temporal changes in the radial and poloidal pressure profile are shown in Figs. 5 and 6, respectively. Since 


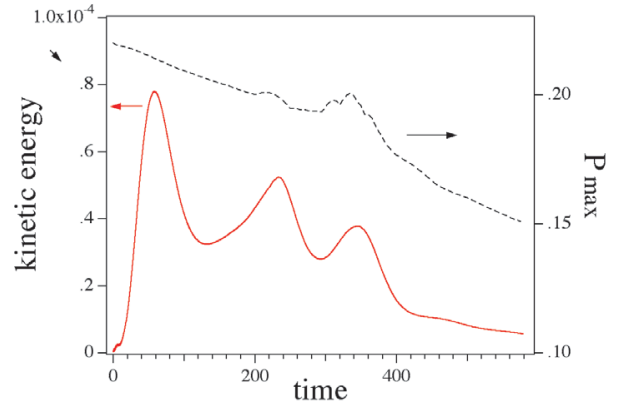

Fig. 4 Time evolution of the total kinetic energy (solid) and the maximum pressure (broken).

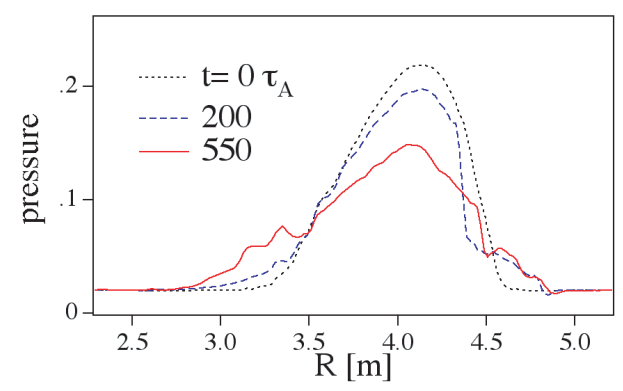

Fig. 5 Temporal change of the radial pressure profile on the horizontally elongated cross section.

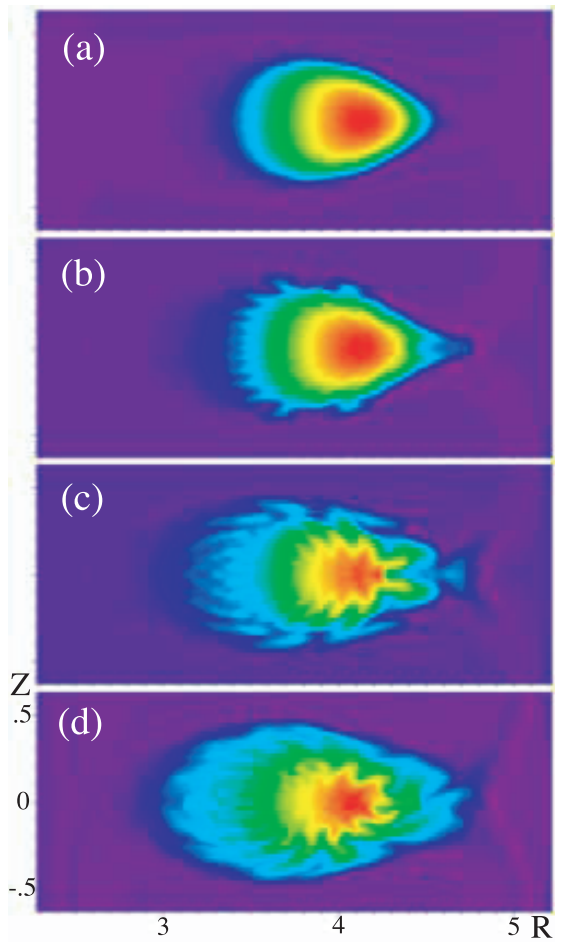

Fig. 6 Temporal change in the pressure profile. The iso-contour maps of the pressure on the horizontally elongated cross section are drawn. The time equals to (a) 0 , (b) 130, (c) 300 , and (d) $550 \tau_{\mathrm{A}}$.

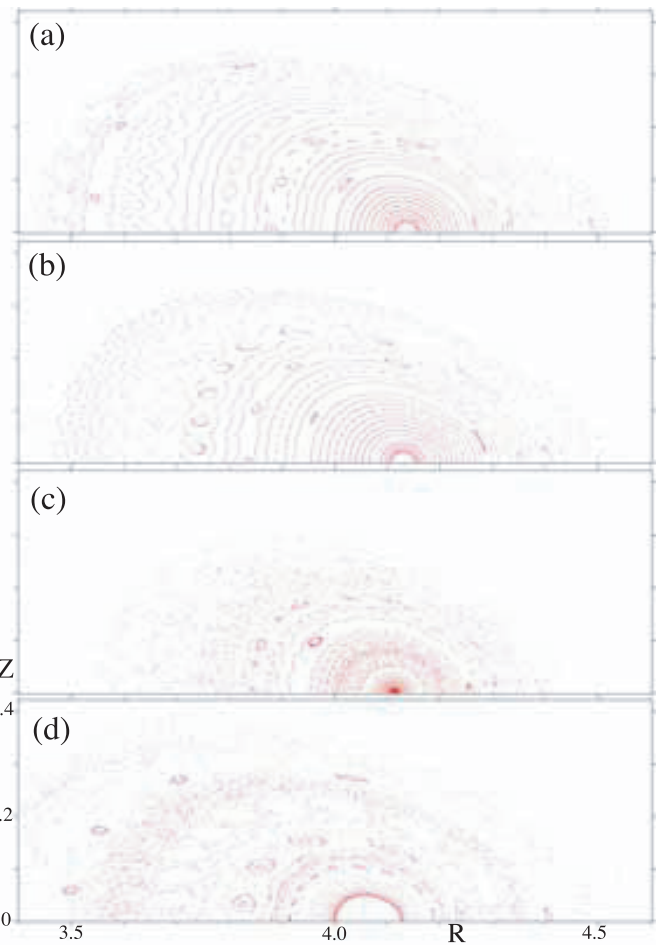

Fig. 7 Temporal change of the magnetic field. The puncture plots of the magnetic field lines on the horizontally elongated cross section are plotted. The time equals to (a) 0 , (b) 130 , (c) 300 , and (d) $550 \tau_{\mathrm{A}}$.

the primarily induced instability is localized in the outer region of the torus, as described above, the crash of the structures is first seen in the barrier region (see Fig. 6(b)). The plasma surface is deformed, reflecting linear eigenmode structures in the outer region and the magnetic field structure in the inner region, as shown in Fig. 3. Part of plasma is lost due to disturbance. The resultant pressure gradient in the barrier region becomes steeper than in the initial state (see Fig. 5 at $t=200 \tau_{\mathrm{A}}$ ). It should be noted that the mode structures of the instability are located only in the barrier region, whereas the central pressure decreases gradually as the lost plasma forms a pedestal pressure in the edge region, as shown in Fig. 5 toward $t=550 \tau_{\mathrm{A}}$ and Figs. 6(b)-(d). If one sees the time development of the maximum pressure at the core as shown in Fig. 4, there is an abrupt change in the trace after $t=300 \tau_{\mathrm{A}}$. The rapid fall in the core pressure might be related to the change in the magnetic field structure. Figure 7 shows the time development of the magnetic field structure, which is expressed by the puncture plot of the field lines. The field line trace is obtained by a sixth-order Runge-Kutta method, together with a fourth-order interpolation of the magnetic field. The magnetic surface structure is clearly formed entirely from the core to the edge region initially (see Fig. 7(a)). Such a nested-surface structure is maintained during the early stage of the relaxation process, although the edge structure is markedly deformed, as shown in Fig. 7(b). At time $t=300 \tau_{\mathrm{A}}$ (see Fig. 7(c)), the magnetic surface structure 
diminishes abruptly throughout the whole poloidal cross section. Finally, the structure reappears in the core region as shown in Fig. 7(d). At the moment shown in Fig. 7(c), the plasma in the core region at high pressure is linked to the external low-pressure region with an identical field line. Under this situation, outward plasma flows due to the pressure imbalances along the field lines, which might cause a rapid fall of the core pressure, can be induced.

The system reaches a relaxed state within $\sim 600 \tau_{\mathrm{A}}$ in this result. The resultant pressure profile becomes broader because of the decrease in the core and the spread in the edge, as shown in Fig. 5 at $t=550 \tau_{\mathrm{A}}$.

\section{Discussion}

The simulation result described in Sec. 3 can be compared qualitatively with the experimental observations on the CDC event in LHD. The time scale of the whole simulation process agrees roughly with that of the crash phase of CDCs. As described in Sec. 1, several questions about the mechanism of occurrence of CDCs are not answered. Our simulation results might provide physical models for them. First, the reason why the core density decreases is a basic question for CDCs. The fact that only the density decreases, keeping the core temperature unchanged, implies that the collapse is governed not by conductive processes, but by convective ones. In our simulation result, the core plasma is extracted convectively through the transiently disordered or reconnected field lines, as shown in Fig. 7. This convective loss mechanism is applicable even if no significant unstable mode exists in the core region. The possibility of the coexistence of the instability in the barrier region and the significant drop in the core pressure is also comparable with another question of why the edge fluctuation is observed just before the CDCs in the experiment. The simulation result provides a reasonable scenario for it. A more detailed comparison would need systematic scans for the edge stability problem both in the simulation and experiment in future.

Now we compare the simulation result with the previous simulation, which studied the nonlinear dynamics of ST plasma on an ELM crash [6]. Although both simulations are initiated by the ballooning mode instability and have several points in common, there is also a qualitative difference between them. To compare both simulations would help us understand the key properties of the process. In Ref. [6], the crash process of the pressure profile is divided in two stages. The edge region is perturbed in the former stage whereas the core pressure falls in the latter one, as is also observed in our simulation. However, for the ST case, the core collapse is caused by a secondary induced internal low- $n$ instability, where $n$ is the toroidal mode number, in contrast to the direct parallel loss mechanism in the helical case described in Sec. 3. No prominent secondary low- $n$ structure can be observed in the helical case simulation, as shown in Fig. 8. The resultant temporal

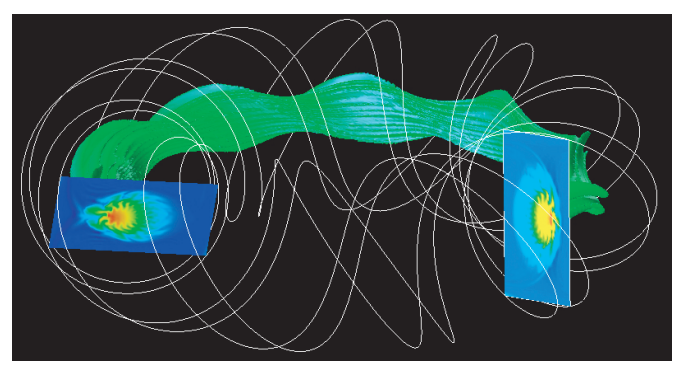

Fig. 8 Three-dimensional structure of plasma pressure at $t=$ $365 \tau_{\mathrm{A}}$. The iso-contour of the pressure in the poloidal cross section and an iso-pressure surface in three dimensions are drawn. The boundary of the simulation region is indicated by white lines.

change of the core pressure profile shows milder activity for the helical case than for the ST case. The large internal plasma current of the ST would cause a difference in the core instability from the helical system, because the induced internal mode is a current driven one.

\section{Summary}

We conducted MHD simulations and revealed the nonlinear dynamics of the ballooning mode in helical systems with a large pressure gradient. The simulation result has shown a physical mechanism that a pressure collapse in the barrier region is followed by a convective loss from the core region through the disturbance of the magnetic field. The simulation is qualitatively comparable with the experimental observations of the CDC events in LHD. Although the simulation result shows a closed scenario initiated by the ballooning instability, it is necessary to conduct the systematic stability analyses under the experimental situation before discussing the cause of the CDC in future.

The authors would like to thank Drs. S. Ohdachi, J. Miyazawa, R. Sakamoto, Y. Narushima, K. Itoh, K. Ichiguchi, Y. Todo, H. Miura and N. Nakajima for fruitful discussion. This work is performed with the support and under the auspices of the NIFS Collaborative Research Program (NIFS07KNXN097/NIFS06KLHH303/ NIFS06KTAT023).

[1] N. Ohyabu, T. Morisaki, S. Masuzaki et al., Phys. Rev. Lett. 97, 55002 (2006).

[2] R. Sakamoto et al., Proc. of Joint Conf. 17th Int. Toki Conf. 16th Int. Stellarator/Heliotron Workshop 2007, Oct. 15-19, 2007, Toki, (2007).

[3] J. Miyazawa et al., Proc. of Joint Conf. 17th Int. Toki Conf. 16th Int. Stellarator/Heliotron Workshop 2007, Oct. 15-19, 2007, Toki, (2007).

[4] K. Hatafuji, T. Hayashi and T. Sato, J. Comp. Phys. 81, 169 (1989).

[5] Y. Suzuki, N. Nakajima, K. Watanabe et al., Nucl. Fusion 46, L19 (2006).

[6] N. Mizuguchi, R. Khan, N. Nakajima and T. Hayashi, Nucl. Fusion 47, 579 (2007). 